\title{
Enhanced osteoblast adhesion on nanostructured selenium compacts for anti-cancer orthopedic applications
}

\author{
Phong Tran' \\ Thomas J Webster ${ }^{2}$ \\ 'Physics Department; ${ }^{2}$ Division of \\ Engineering and Department of \\ Orthopedics, Brown University, \\ Providence, USA
}

\begin{abstract}
Metallic bone implants possess numerous problems limiting their long-term efficacy, such as poor prolonged osseointegration, stress shielding, and corrosion under in vivo environments. Such problems are compounded for bone cancer patients since numerous patients receive orthopedic implants after cancerous bone resection. Unfortunately, current orthopedic materials were not originally developed to simultaneously increase healthy bone growth (as in traditional orthopedic implant applications) while inhibiting cancerous bone growth. The long-term objective of the present research is to investigate the use of nano-rough selenium to prevent bone cancer from re-occurring while promoting healthy bone growth for this select group of cancer patients. Selenium is a well known anti-cancer chemical. However, what is not known is how healthy bone cells interact with selenium. To determine this, selenium, spherical or semispherical shots, were pressed into cylindrical compacts and these compacts were then etched using $1 \mathrm{~N} \mathrm{NaOH}$ to obtain various surface structures ranging from the micron, submicron to nano scales. Changes in surface chemistry were also analyzed. Through these etching techniques, results of this study showed that biologically inspired surface roughness values were created on selenium compacts to match that of natural bone roughness. Moreover, results showed that healthy bone cell adhesion increased with greater nanometer selenium roughness (more closely matching that of titanium). In this manner, this study suggests that nano-rough selenium should be further tested for orthopedic applications involving bone cancer treatment.
\end{abstract}

Keywords: selenium, nano-rough, osteoblast, cancer, chemopreventive

\section{Introduction}

The most commonly used materials for bone implants are metals (such as titanium, Ti) and polymers (such as ultra high molecular weight polyethylene). Numerous problems exist with these implants such as (i) insufficient prolonged bonding between the implanted material and juxtaposed bone (Kaplan et al 1994a, 1994b; Buser et al 1999; Webster 2001), (ii) different mechanical properties between bone and the implant leading to stress shielding (Kaplan et al 1994a, 1994b; Webster 2001), and (iii) wear debris generated at articulating surfaces of orthopedic implants that may lead to cell death (Kaplan et al 1994a, 1994b). It has been speculated that nano-structured materials can increase orthopedic implant efficacy since it is well known that the nanometer scale is the length scale that most of the body's natural materials possess. For example, hydroxyapatite, the major inorganic component of bone, exists predominantly as platelet-or rod-shape crystals about $2-5 \mathrm{~nm}$ in width and $50 \mathrm{~nm}$ in length. Type I collagen, the major organic component of bone, has fibrils $300 \mathrm{~nm}$ in length, $0.5 \mathrm{~nm}$ in width, and has a periodicity of $67 \mathrm{~nm}$ (Park and Lakes 1992). Therefore, it is reasonable to consider that osteoblasts (or bone-forming cells) will be more accustomed to nano-structured surfaces compared to currently used nano-smooth implant surfaces. 
Such problems with promoting new bone growth next to implant surfaces are made only more complex for patients with bone cancer (both primary bone cancer and metastasized bone cancers). For example, it is estimated that 2,380 people will be diagnosed with bone and joint cancers and 1,470 people will die from primary bone and joint cancers in 2008 in the US (American Cancer Society 2008). Primary bone cancer is rare but bone cancer as a result of the metastasis from other organs (such as the lungs, breasts and the prostate) is very common (Miller and Webster 2007). Thus, the number of patients affiliated with bone cancer is much higher when considering the statistics of cancer metastasis. For these reasons, the main goal of this study was to introduce a new biomaterial naturally found in the human body that has chemopreventive properties for orthopedic implants: elemental selenium. The first goal in this effort was to create nano-structured roughness on such materials and to determine bone cell responses on such nano-structured selenium materials.

\section{Materials and methods}

Selenium shots (amorphous, metals basis, spherical and/or semi-spherical 2-4 mm in diameter; Alfa Asear, Ward Hill, MA) were pressed into cylindrical compacts $(0.635 \mathrm{~cm}$ radius and $0.2 \mathrm{~cm}$ thickness) at 1000 psi for 2 minutes using a uniaxial compacting hydraulic press (Carver, Inc., Wabash, IN). The compacts were then chemically treated with $1 \mathrm{~N} \mathrm{NaOH}$ for either 10 or $30 \mathrm{~min}$. After etching, selenium compacts were washed with excessive deionized water to remove the $\mathrm{NaOH}$ that remained on the selenium compacts. Titanium substrates (Alfa Aesar) were used as controls.

Surfaces of untreated selenium compacts as well as selenium compacts treated with $1 \mathrm{~N} \mathrm{NaOH}$ for 10 and 30 min were visualized (without a conductive coating) using a scanning electron microscope (SEM, LEO 1530VP FE-4800) with an accelerating voltage from 3 to $10 \mathrm{kV}$. Energy dispersive
X-ray spectroscopy (EDS, LEO 1530) was used to determine the surface chemistry of the compacts.

To investigate osteoblast adhesion on selenium compacts, human osteoblast-like cells (bone-forming cells; CRL-11372 American Type of Culture Collection, population numbers 14-15) in Dulbecco's Modified Eagle Media supplemented with 10\% fetal bovine serum (Hyclone) and 1\% Penicillin/ Streptomycin (Hyclone) were seeded at a density of 3500 cells $/ \mathrm{cm}^{2}$ and placed in an incubator under standard cell culture conditions $\left(37^{\circ} \mathrm{C}, 5 \% \mathrm{CO}_{2}, 95 \%\right.$ humidified air) for $24 \mathrm{~h}$. Importantly, this is an immortalized cell line which has been used widely in experiments involving orthopedic applications since previous studies have demonstrated that when cultured under similar conditions as described in the present study, these cells secrete bone-related proteins and deposit calcium containing mineral (Miller and Webster 2007). The cells were then fixed using 4\% formaldehyde (Sigma, St. Louis, MO), stained with 4',6-diamidino-2-phenylindole (DAPI) (Sigma) and counted under fluorescence microscopy (Zeiss Axiovert 200M Light Microscope) in five random fields which were averaged for each compact. Cell experiments were conducted in triplicate and repeated three times. Data were collected and statistical comparisons were assessed using a one-tailed Student t-test.

\section{Results and discussion}

The SEM images of the selenium compacts (untreated and treated with $\mathrm{NaOH}$ for different time periods) revealed various surface roughness. Untreated surfaces (Figure 1 image A) possessed mostly micron rough surface features while those treated with $1 \mathrm{~N} \mathrm{NaOH}$ for different time periods created submicron (Figure 1 image B) and nano-rough (Figure 1 image $C$ ) surface features on selenium. In particular, biologically inspired surface roughness increased with increasing $\mathrm{NaOH}$ treatment time periods. As the etchant, $\mathrm{NaOH}$ is believed to dissolve the oxide layer on the surface

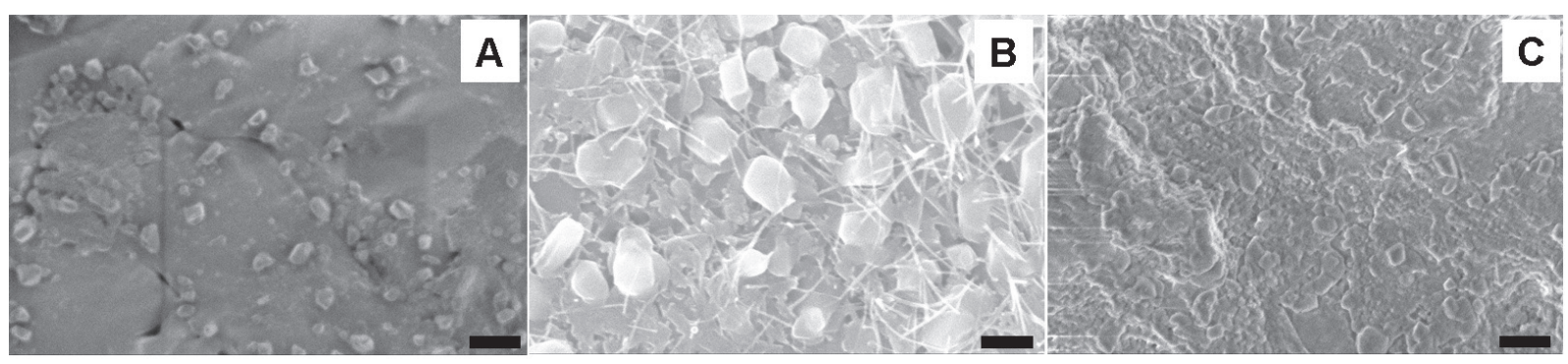

Figure I SEM images of an untreated selenium compact (A) and selenium compacts treated with IN NaOH for 10 min (B) and 30 min (C). Note: Bars $=1$ micrometer. 
of the selenium compacts creating submicron and nanometer features at increasing times.

Chemical etching is a very common, inexpensive, and simple method used to modify the surface properties (eg, topography, chemistry, roughness, wettability, etc.) of biomaterials. Chemical etching can be used for a wide range of biomaterials from metals, ceramics, to polymers. For example, etching titanium with $\mathrm{HCl}$ followed by $\mathrm{NaOH}$ has led to the formation of uniform micrometer featured surfaces that increased the formation of hydroxycarbonated apatite when exposed to a simulated body fluid (Jonasova et al 2004). Moreover, etching poly (lactic-co-glycolic acid) (PLGA) films using $\mathrm{NaOH}$ has created biologically inspired nanometer surface feature dimensions to promote the functions of numerous cells. For example, this nanometer PLGA structured surface was shown to promote bladder smooth muscle cell functions compared to conventional, micrometer-structured PLGA after 1, 3, and 5 days of culture (Thapa et al 2003). In addition, Feldspathic porcelain etched in $5 \%$ hydrofluoric acid was shown to promote the bonding strength between orthodontic brackets and ceramic crowns when compared to those treated by mechanical roughening with fine diamond burs or sandblasting (Schmage et al 2003). Clearly, chemical etching can be an inexpensive effective way to transform nano-smooth surfaces into nano-rough surfaces for a wide range of materials to promote their biological performance.

Importantly, EDS profiles of the compacts used in the present study revealed that the chemistry of the selenium compacts remained unaffected after treatment in $1 \mathrm{~N} \mathrm{NaOH}$ (Figure 2). Moreover, Table 1 lists the weight percentages of selenium and sodium for each compact. The results showed trace amounts of sodium on the surfaces of selenium compacts treated with $1 \mathrm{~N} \mathrm{NaOH}$. This indicated that chemical etching (ie, using $1 \mathrm{~N} \mathrm{NaOH}$ for up to $30 \mathrm{~min}$ ) only changed the surface roughness, not the surface chemistry of the selenium compacts. The ability to change an implant's surface roughness and/or topography without altering its chemistry is of great interest since, when fabricating implant materials, it is crucial not to induce any potential harmful chemistry changes. In this study, selenium compact chemistry remained unaltered after treatment with $1 \mathrm{~N} \mathrm{NaOH}$ for up to $30 \mathrm{~min}$.

When these substrates were used as substrates for culturing osteoblasts, after 1 day, significantly increased cell densities were observed on the increasing nanorough surfaces (ie, compacts treated with $1 \mathrm{~N} \mathrm{NaOH}$ for 10 and $30 \mathrm{~min}$ ) (Figures 3, 4). Especially, the compacts treated with $1 \mathrm{~N} \mathrm{NaOH}$ for $30 \mathrm{~min}$, which had the largest amount of nanometer surface features, had the highest osteoblast density. However, although closer, these selenium compacts (ie, compacts treated with $1 \mathrm{~N} \mathrm{NaOH}$ for $30 \mathrm{~min}$ ) had osteoblast densities lower than that of titanium. Future studies will need to utilize greater times and/or concentrations of $\mathrm{NaOH}$ etching to determine if osteoblast adhesion can be matched between selenium and titanium. Moreover, future studies will need to examine primary osteoblast cells (since that was not accomplished here) as well as the multitude of cancerous cells that can cause bone cancer.

It has been shown that increased nanometer surface roughness promotes osteoblast functions (from adhesion to the deposition of calcium containing mineral) (Webster et al 1999, 2000a, 2000b; Webster 2001; Webster and Ejiofor 2004). The results of this study showed a similar trend of increased healthy osteoblast densities after 1 day of culture on selenium with increased nanometer surface roughness.

The significance of these results is also the introduction of a new chemistry to the orthopedic community particularly geared at inhibiting bone cancer regrowth: selenium. Selenium has been shown to have chemopreventive effects in numerous reports (Combs and Combs 1986; Clark et al 1991, 1998; Combs and Gray 1998; Navarro-Alarcon and Lopez-Martinez 2000; Zhuo et al 2004; Wei et al 2004). However, the mechanisms of selenium-based chemoprevention are complex and incompletely understood (Combs 2001). By imparting nano features onto selenium compacts, the objective of this study was to create a material that promotes healthy osteoblast functions since evidence has already been provided concerning its role in inhibiting cancer growth. In the present study, healthy osteoblast adhesion was shown to be enhanced on nano-rough selenium compacts when compared to micro-rough selenium compacts. The reasons why nano-roughness enhances osteoblast functions remains incompletely understood. However, it has been shown that nano-roughness effectively increased the initial absorption of proteins (such as fibronectin and vitronectin) which mediate subsequent cell adhesion (Webster et al 2000b, 2001; Khang et al 2007). In particular, particle boundaries on other material (Ti, Ti6Al4V, and CoCrMo) surfaces were shown to be active regions for such protein adsorption (Webster and Ejiofor 2004). Nano-structured surfaces clearly offer more particle boundaries when compared to micron-structured surfaces for promoting initial protein adsorption events. 

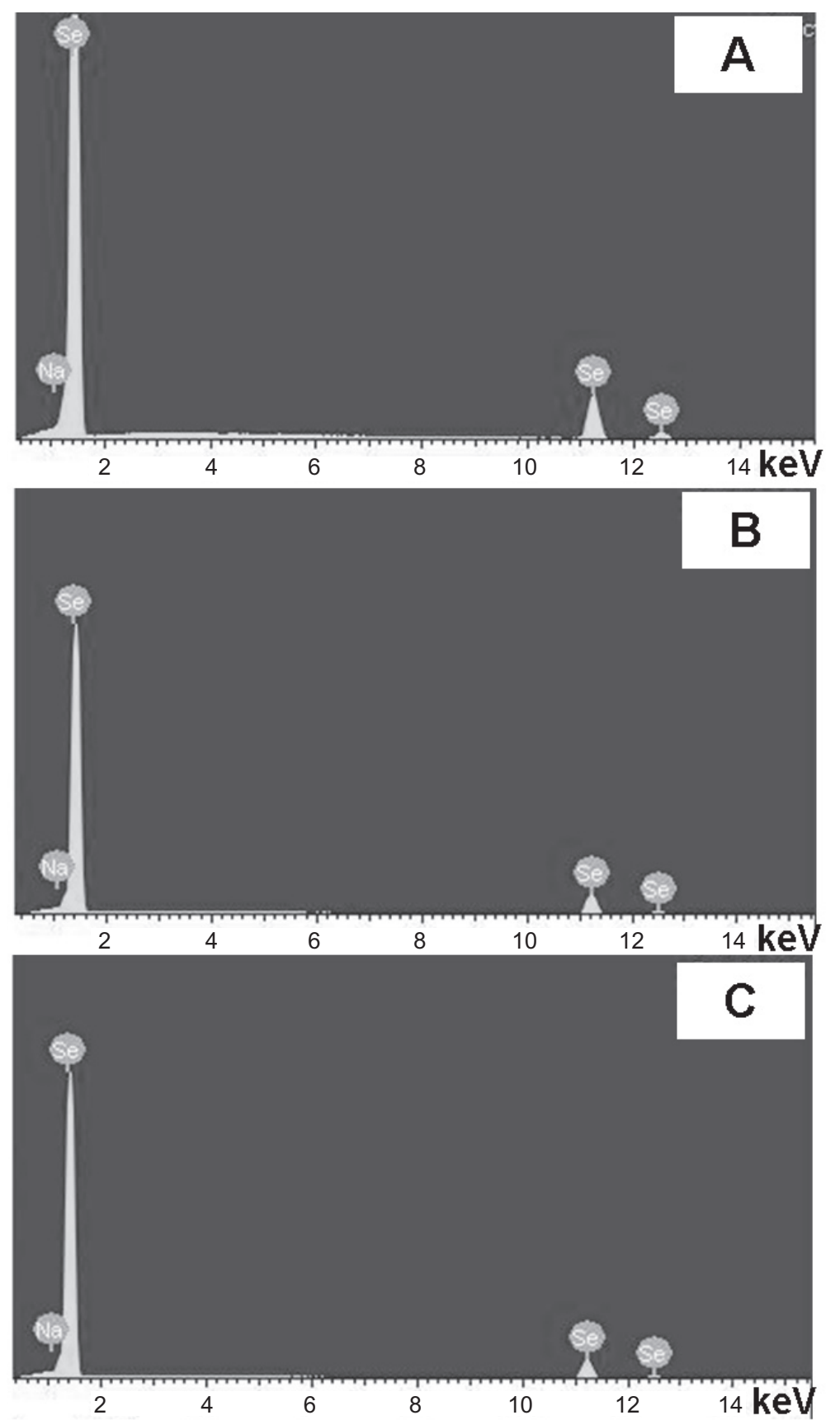

Figure 2 EDS profiles of (A) untreated selenium compacts and (B) selenium compacts treated with IN NaOH for 10 min and (C) 30 min.

Table I Weight percentages of selenium (Se) and $\mathrm{Na}$ on Se compact surfaces*

\begin{tabular}{lll}
\hline Se compacts & Weight \% of Se & Weight \% of $\mathbf{~ N a}$ \\
\hline Untreated & 99.94 & 0.06 \\
Treated with IN NaOH & 99.82 & 0.18 \\
for 10 min & & \\
Treated with IN NaOH & 99.72 & 0.28 \\
for 30 min & & \\
\hline
\end{tabular}

Note: *Data not statistically different.
In another recent study, elemental selenium nanoclusters were coated on conventional titanium substrates to create nano-structured surfaces. These surfaces were shown to promote healthy osteoblast functions after 1 day of culture and, importantly, inhibit cancerous osteoblast cell functions after 3 days of culture (Sarin et al 2008). In the present study, nano-structured selenium surfaces were created by a different method: chemically etching selenium bulk compacts. The approach used in this study is simpler than the coating method 

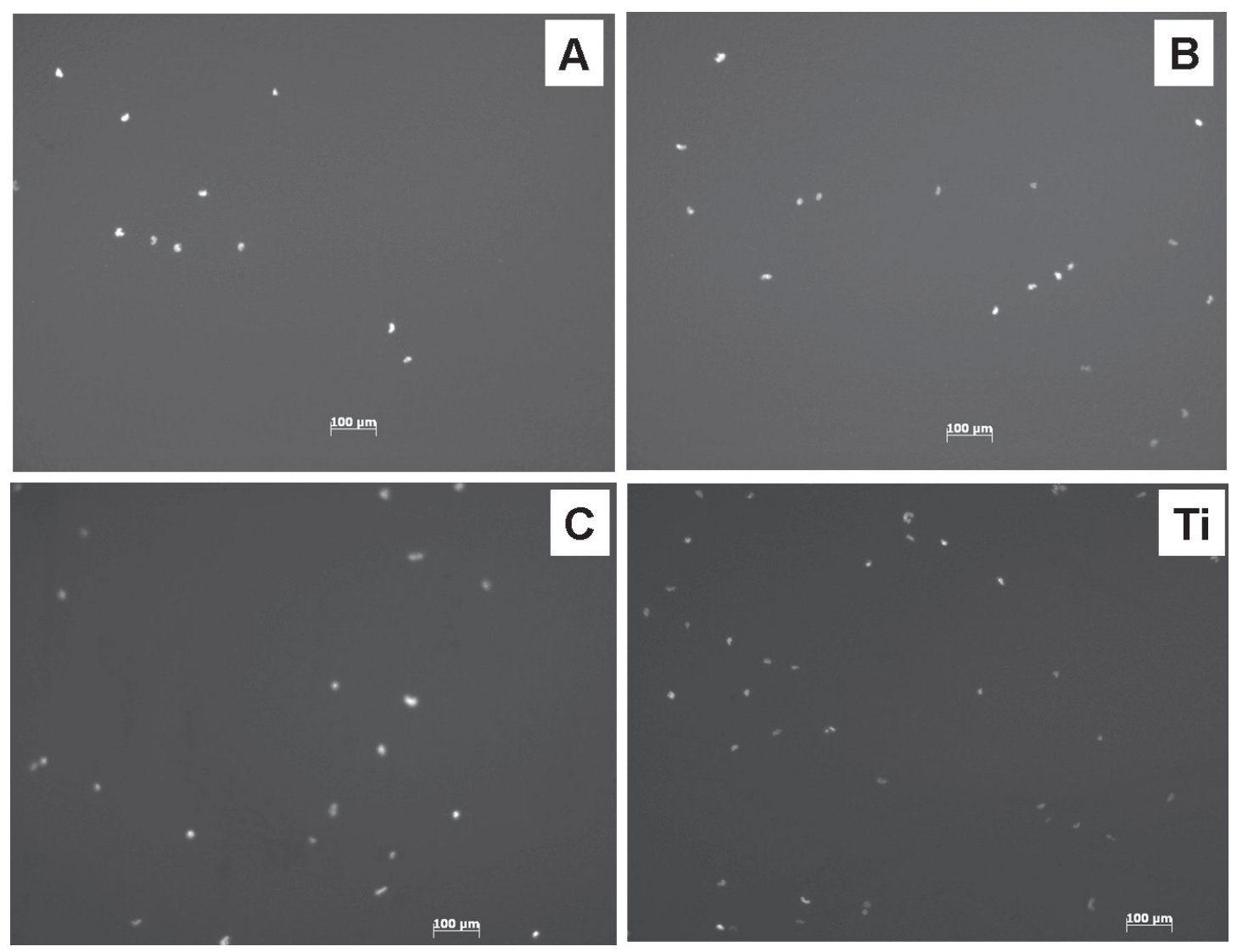

Figure 3 Fluorescence microscopy images of osteoblasts on: (A) Untreated selenium compacts; (B) selenium compacts treated with IN NaOH for 10 min; (C) selenium compacts treated with IN NaOH for 30 min; and Titanium (Ti) substrates.

Note: Time $=24 \mathrm{~h}$

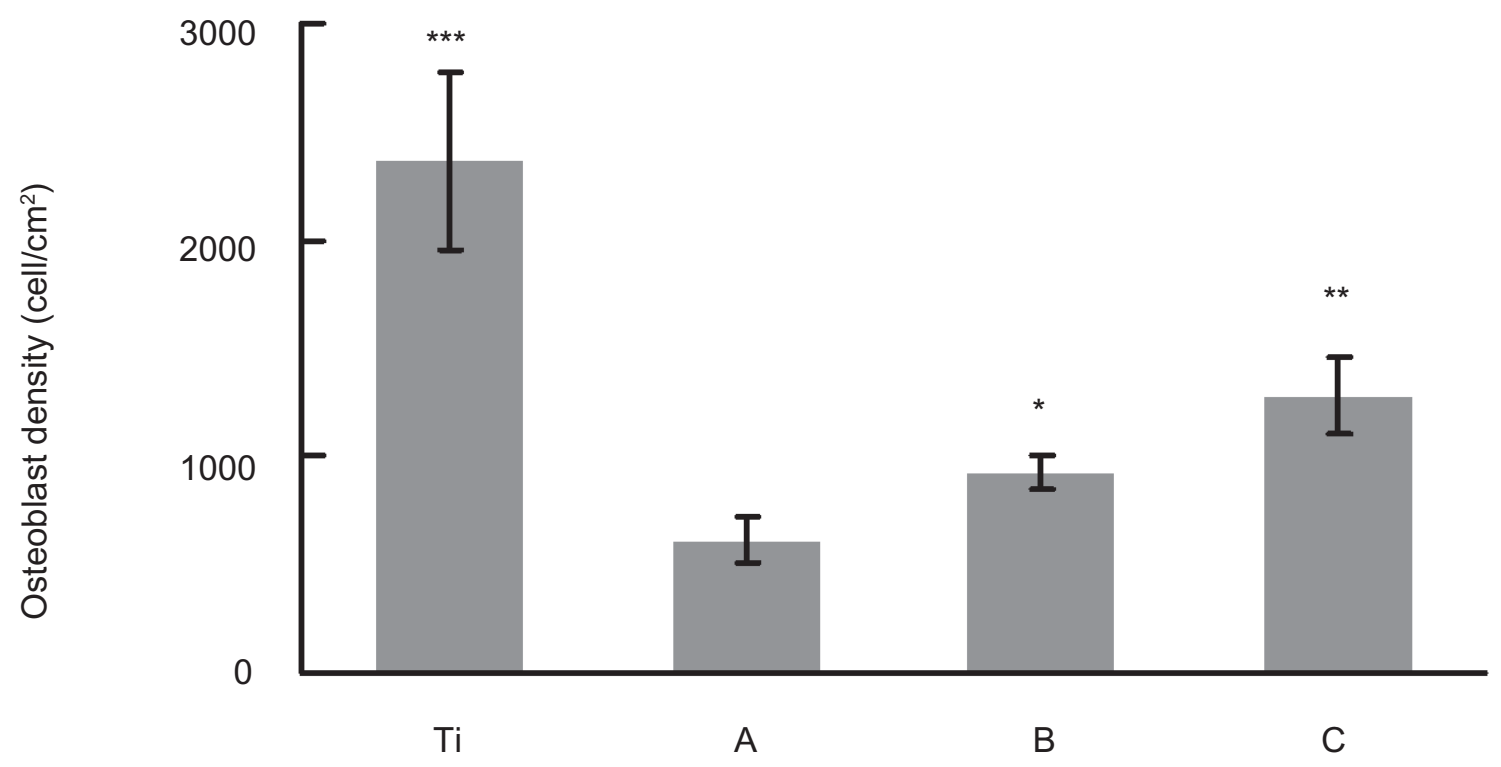

Figure 4 Increased osteoblast densities on selenium compacts with greater amounts of nanosurface features after $24 \mathrm{~h}$ of culturing. (A) Untreated selenium compacts; (B) selenium compacts treated with IN NaOH for 10 min; and (C) selenium compacts treated with IN NaOH for 30 min.

Notes: $\mathrm{Data}=$ Mean $\pm \mathrm{SEM} ; \mathrm{N}=3$; ${ }^{*} \mathrm{p}<0.05$ compared to untreated substrate; ${ }^{* *} \mathrm{p}<0.05$ compared to compacts treated with IN NaOH for I0 min. ${ }^{* * *} \mathrm{p}<0.0 \mathrm{I}$ compared to compacts treated with IN NaOH for $30 \mathrm{~min}$. 
used (which involved selenium chemical precipitation) and can eliminate the influence of titanium in the interaction with osteoblasts since selenium coatings in that previous study did not completely cover the underlying titanium. However, considering the toxicity of selenium at high levels (Whanger et al 1996), the use of selenium compacts may be problematic concerning how much selenium is exposed to the body leading to possible toxic effects. Future studies need to clearly investigate this possible toxicity as well as cancerous bone cell functions on these selenium compacts.

\section{Conclusions}

This study provided techniques to create nano-structured roughness without altering chemistry on selenium compacts for anti-cancer orthopedic applications. Since previous studies have shown greater osteoblast functions on nano-structured compared to conventional ceramics, metals, polymers, and composites, the ability to create nano-structured roughness on selenium compacts is promising for increasing bone cell functions, while inhibiting the return of bone cancer.

\section{Acknowledgments}

The authors would like to thank Mr. Anthony McCormick for help with SEM and Mr. Chang Yao for help with cell experiments. The authors would also like to thank the Herman Foundation for funding. The authors report no conflicts of interest in this work.

\section{References}

American Cancer Society. 2008. Cancer facts and figures - 2008 [online]. Accessed 30 April 2008. URL: http://www.cancer.org/docroot/STT/ content/STT_1x_Cancer_Facts_and_Figures_2008.asp.

Buser D, Nydegger I, Oxland I, et al. 1999. Interface shear strength of titanium implants with a sandblasted and acid-etched surface: A biomechanical study in the maxilla of miniature pigs. J Biomed Mater Res, 45:75-82.

Clark LC, Cantor KP, Allaway WH. 1991. Selenium in forage crops and cancer mortality in US counties. Arch Environ Health, 146:37-42.

Clark LC, Dalkin B, Krongrad A, et al. 1998. Decreased incidence of prostate cancer with selenium supplementation: results of a double-blind cancer prevention trial. Br J Urol, 81:730-4.

Combs GFJ, Combs SB. 1986. The role of selenium in nutrition. Orlando, FL: Academic Press.

Combs GFJ, Gray WP. 1998. Chemopreventive agents: selenium. Pharmacol Ther, 79:179-92.
Combs GF. 2001. Impact of selenium and cancer-prevention findings on the nutrition-health paradigm. Nutr Cancer, 40:6-11.

Jonasova L, Muller FA, Helebrant A, et al. 2004. Biomimetic apatite formation on chemically treated titanium. Biomaterials, 25:1187-94.

Kaplan FS, Hayes WC, Keaveny TM, et al. 1994a. Biomaterials. In: Simon SP (ed). Orthopedic Basic Science. Columbus, OH: American Academy of Orthopedic Surgeons. p. 460-78.

Kaplan FS, Lee WC, Keaveny TM, et al. 1994b. Forms and functions of bone. In: Simon SP (ed). Orthopedic basic science. Columbus, $\mathrm{OH}$ : American Academy of Orthopedic Surgeons. p. 127-85.

Khang D, Kim SY, Liu-Snyder P, et al. 2007. Enhanced fibronectin adsorption on carbon nanotube/poly(carbonate) urethane: Independent role of surface nano-roughness and associated surface energy. Biomaterials, 28:4756-68.

Miller D, Webster TJ. 2007. Anticancer orthopedic implants. In: Nalwa H, Webster TJ (ed). Cancer nanotechnology. California: American Scientific. P. 307-16.

Navarro-Alarcon M, Lopez-Martinez MC. 2000. Essentiality of selenium in the human body: relationship with different diseases. Sci Total Environ, 249:347-71.

Park JB, Lakes RS. 1992. Biomaterials - An introduction. New York: Plenum Press.

Sarin L, Tran P, Hurt R, et al. 2008. Increased healthy osteoblast and decreased cancerous osteoblast density on selenium coated on titanium. $J$ Biomed Mater Res, In press.

Schmage P, Nergiz I, Herrmann W, et al. 2003. Influence of various surface-conditioning methods on the bond strength of metal brackets to ceramic surfaces. Am J Orthod Dentofacial Orthop, 123:540-6.

Thapa A, Miller DC, Webster TJ, et al. 2003. Nano-structured polymers enhance bladder smooth muscle cell function. Biomaterials, 24:2915-26.

Webster TJ, Siegel RW, Bizios R. 1999. Osteoblast adhesion on nanophase ceramics. Biomaterials, 20:1221-4.

Webster TJ, Siegel RW, Bizios R. 2000a. Enhanced functions of osteoblasts on nanophase ceramics. Biomaterials, 21:1803-9.

Webster TJ, Ergun C, Doremus RH, et al. 2000b. Specific proteins mediate enhanced osteoblast adhesion on nanophase ceramics. J Biomed Mater Res, 51:475-80.

Webster TJ. 2001. Nanophase ceramics: the future of orthopedic and dental implant material. In: Ying JY (ed). Nanostructured Materials. New York: Academy Press. P. 125-66.

Webster TJ, Schadler LS, Siegel RW, et al. 2001. Mechanisms of enhanced osteoblast adhesion on nanophase alumina involve vitronectin. Tissue Eng, 7:291-301.

Webster TJ, Ejiofor JU. 2004. Increased osteoblast adhesion on nanophase metals: Ti, Ti6Al4V, and CoCrMo. Biomaterials, 25:4731-9.

Wei WQ, Abnet CC, Qiao YL, et al. 2004. Prospective study of serum selenium concentrations and esophageal and gastric cardiac cancer, heart disease, stroke and total death. Am J Clin Nutr, 79:80-5.

Whanger P, Vendeland S, ParkYC, et al. 1996. Metabolism of subtoxic levels of selenium in animals and humans. Ann Clin Lab Sci, 26:99-113.

Zhuo H, Smith AH, Steinmaus C. 2004. Selenium and lung cancer: a quantitative analysis of heterogeneity in the current epidemiological literature. Cancer Epidemiol Biomarkers Prev, 13:771-8. 
